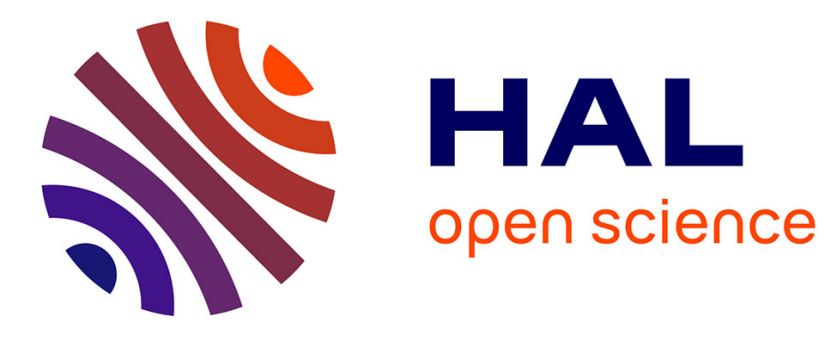

\title{
The Degree Ratio Ranking Method for Directed Networks
}

René van den Brink, Agnieszka Rusinowska

\section{To cite this version:}

René van den Brink, Agnieszka Rusinowska. The Degree Ratio Ranking Method for Directed Networks. 2019. halshs-02143874

\section{HAL Id: halshs-02143874 \\ https://shs.hal.science/halshs-02143874}

Submitted on 29 May 2019

HAL is a multi-disciplinary open access archive for the deposit and dissemination of scientific research documents, whether they are published or not. The documents may come from teaching and research institutions in France or abroad, or from public or private research centers.
L'archive ouverte pluridisciplinaire $\mathbf{H A L}$, est destinée au dépôt et à la diffusion de documents scientifiques de niveau recherche, publiés ou non, émanant des établissements d'enseignement et de recherche français ou étrangers, des laboratoires publics ou privés. 


\section{Documents de Travail du

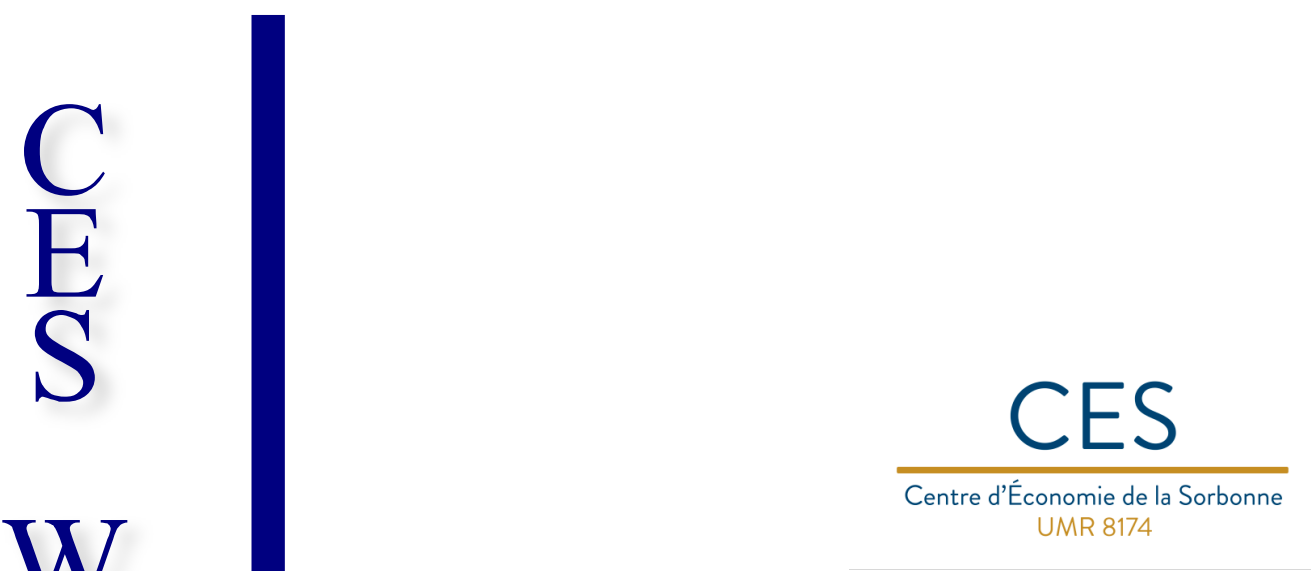

The Degree Ratio Ranking Method for Directed Networks

René VAN Den BRINK, Agnieszka RusinowsKA

2019.09

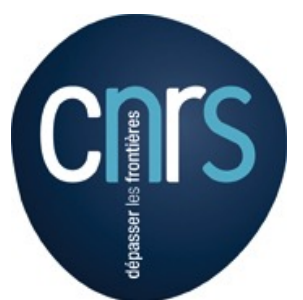

Maison des Sciences Économiques, 106-112 boulevard de L'Hôpital, 75647 Paris Cedex 13 


\title{
The Degree Ratio Ranking Method for Directed Networks ${ }^{\star}$
}

\author{
René van den Brink ${ }^{\star \star 1}$ and Agnieszka Rusinowska ${ }^{2}$ \\ 1 Department of Econometrics and Operations Research, and Tinbergen Institute, VU University \\ De Boelelaan 1105, 1081 HV Amsterdam, The Netherlands \\ jrbrink@feweb.vu.nl \\ 2 CNRS, Paris School of Economics, Centre d'Economie de la Sorbonne \\ 106-112 Bd de l'Hôpital, 75647 Paris Cedex 13, France \\ agnieszka.rusinowska@univ-paris1.fr
}

\begin{abstract}
One of the most famous ranking methods for digraphs is the ranking by Copeland score. The Copeland score of a node in a digraph is the difference between its outdegree (i.e. its number of outgoing arcs) and its indegree (i.e. its number of ingoing arcs). In the ranking by Copeland score, a node is ranked higher, the higher is its Copeland score. In this paper, we deal with an alternative to rank nodes according to their out- and indegree, namely ranking the nodes according to their degree ratio, i.e. the outdegree divided by the indegree. To avoid dividing by a zero indegree, we implicitly take the out- and indegree of the reflexive digraph. We provide an axiomatization of the ranking by degree ratio using a sibling neutrality axiom, which says that the entrance of a sibling (i.e. a node that is in some sense similar to the original node) does not change the ranking among the original nodes. We also provide a new axiomatization of the ranking by Copeland score using the same axioms except that this method satisfies a different sibling neutrality. Finally, we modify the ranking by degree ratio by not considering the reflexive digraph, but by definition assume nodes with indegree zero to be ranked higher than nodes with a positive indegree. We provide an axiomatization of this ranking by modified degree ratio using yet another sibling neutrality and a maximal property. In this way, we can compare the three ranking methods by their respective sibling neutrality.
\end{abstract}

JEL Classification: D71, D85, C02

Keywords: group decisions and negotiations, directed graph, ranking method, degree ratio, Copeland score

\footnotetext{
* This research has been initiated when René van den Brink was Visiting Professor at the Centre d'Economie de la Sorbonne of the University of Paris 1. Agnieszka Rusinowska acknowledges the support by the National Agency for Research (Agence Nationale de la Recherche) - Project "CoCoRICo-CoDec" (ANR-14-CE24-0007-01).

** Corresponding author
} 


\section{Introduction}

Ranking is one of the crucial issues in everyday economic, social, and political activities. Ranking departments and candidates within a university, journals (Pinski and Narin (1976)), web pages on the internet (Brin and Page (1998)), teams in sport competitions, firms in an industry, political candidates and alternatives in social choice (Sen (1979)), these are just some of numerous examples. The use of an appropriate ranking method is of particular importance. Directed graphs have the established role in modeling and investigating various ranking methods, with nodes having different interpretations (individuals, journals, web pages, teams, alternatives, institutions, political parties, etc.).

Formally, a ranking method assigns to every directed graph a complete preorder on the set of nodes. One of the most famous ranking methods for digraphs is the ranking by Copeland score (Copeland (1951)). The Copeland score of a node in a digraph is the difference between its outdegree (i.e. its number of outgoing arcs) and its indegree (i.e. its number of ingoing arcs). In the ranking by Copeland score, a node is ranked higher, the higher is its Copeland score. We investigate an alternative to rank nodes according to their out- and indegree, namely ranking the nodes according to their degree ratio, i.e. the outdegree divided by the indegree. In some types of situations the degree ratio ranking can give different results from the ranking by Copeland score. While a draw (i.e., two arcs between the same nodes oriented in opposite direction) is neutral in the Copeland score, it is not in our ranking by the degree ratio. In particular, when an agent loses more (respectively less) frequently than gains, a draw is beneficial (respectively disadvantageous) for such an agent. Therefore, when losing by itself is seen more negatively than winning is seen positively, the degree ratio ranking might be a more appropriate ranking method to use.

The degree ratio ranking method can be applied in various fields. In fact, it is applied in ranking players in online video gaming ${ }^{1}$. When the nodes in a digraph represent countries (or regions) and the arcs represent migration flows, then the degree ratio gives for each country its emigration relative to (i.e. divided by) its immigration, which can be seen as an alternative to the usual net migration rate, being essentially a Copeland score. In case the digraph represents a financial network where outgoing arcs represent money borrowed to other banks, and ingoing arcs represent money borrowed from other banks, the degree ratio of a bank is its debit to credit ratio. A similar interpretation can be given when the digraph represents a mutual control network of firms investing in each other.

To start the analysis of the ranking by degree ratio, we first define the degree ratio by implicitly taking the out- and indegree of the reflexive digraph. We use the reflexive digraph to avoid dividing by (indegree) zero. We provide an axiomatization of the ranking by degree ratio using a sibling neutrality, which says that the entrance of a sibling of a node (i.e. a node that is similar to the original node) does not change the ranking between this node and the other original nodes. Other properties that, together with sibling neutrality, characterize the ranking by degree ratio are anonymity, positive responsiveness,

\footnotetext{
${ }^{1}$ For example, participants in the popular Fortnite online video game are ranked by the 'Kill to Death ratio' (KDR), which is their degree ratio in the digraph where a player's outgoing arcs are to the opponents that he/she killed, and the ingoing arcs are from the opponents who killed him/her. In the game you can resurrect after being killed, and therefore you can be killed multiple times. The idea behind the KD ratio is that it gives the average number of kills you make on average in a life, which is considered a good measure of the strength of the players.
} 
independence of irrelevant arcs, and the intermediary property. Anonymity means that relabeling the nodes implies a corresponding relabeling in the ranking. According to positive responsiveness, when one of two equally ranked nodes gets one more outgoing arc, this node becomes strictly higher ranked than the other one. Independence of irrelevant arcs implies that, when pairwise comparing two nodes, we only need to consider the arcs that involve these two nodes. The intermediary property means that putting only one new node on an existing arc, does not change the ranking.

Besides characterizing the ranking by degree ratio, we also provide a new axiomatization of the ranking by Copeland score using the same axioms, except that this ranking method satisfies a different sibling neutrality.

Finally, we modify the ranking by degree ratio by not considering the reflexive digraph, but we assume that nodes with indegree zero are ranked higher than nodes with a positive indegree. Obviously, such a ranking by modified degree ratio is different from the ranking by degree ratio and the Copeland score. However, if the degree and modified degree ratios give different ranking of two nodes with positive indegrees, then their ranking by degree ratio is always the same as their ranking by Copeland score. We provide an axiomatic characterization of the ranking by modified degree ratio by similar axioms as mentioned before, except that we replace sibling neutrality by yet another modified sibling neutrality and an additional maximal property. This last axiom requires to rank nodes with zero indegree above nodes with positive indegree. In this sense, this method refines the set of strong maximal nodes, being the set of nodes with indegree zero. If a strong maximal node exists then the node that is ranked highest is a strong maximal node.

Related literature There exists a vast literature that studies the Copeland score as well as other ranking methods. Rubinstein (1980) characterizes the ranking by Copeland score on the class of tournaments. The ranking by Copeland score is also characterized, e.g., by Henriet (1985) and Bouyssou (1992); see also Bouyssou and Perny (1992) for a related ranking method. On the class of tournaments, the ranking by Copeland score coincides with the ranking by outdegree. In van den Brink and Gilles (2003), a generalization of Rubinstein's result is presented by characterizing the ranking by outdegree for arbitrary directed graphs. An axiomatic characterization of the outflow ranking method for weighted directed graphs is provided in van den Brink and Gilles (2009), while van den Brink and Gilles (2000) axiomatize the outflow as a relational power measure for weighted and nonweighted directed graphs. Bouyssou and Marchant (2018) axiomatize the alternative $\beta$ ranking method for directed networks, which is based on the $\beta$-measure introduced in van den Brink and Gilles (2000). Several works study methods based on evaluations or citations and consider one-sided or peers' settings; see e.g., Altman and Tennenholtz (2005), Palacios-Huerta and Volij (2004), Slutzki and Volij (2006). In a one-sided setting, experts provide evaluations on some items to be ranked. We obtain the peers setting when the experts coincide with the items (e.g. Webpages linking to other pages or journals citing each other). Du et al. (2015) study ranking of items in a graph determined by a choice of utility function. Demange (2014a) and Demange (2017) characterizes ranking methods based on evaluations or citations which consider one-sided settings and two-sided settings, respectively. The Hirsch index, one of the ranking methods supporting evaluations of researchers, is axiomatically characterized in Woeginger (2008). For other works considering the peers setting, e.g., incentive compatibility, see Altman and Tennenholtz 
(2008), de Clippel et al. (2008), and for some studies of rankings in a dynamic setting, see Demange (2012) and Demange (2014b).

The underlying paper is structured as follows. In Section 2, we present basic preliminaries on digraphs and ranking methods. The ranking by degree ratio is introduced and axiomatized in Section 3. In Section 4, we provide a comparable axiomatization of the ranking by Copeland score. The ranking by modified degree ratio is introduced and axiomatically characterized in Section 5. We present some concluding remarks in Section 6. All proofs of the main results of the paper are given in the Appendix.

\section{Preliminaries}

A directed graph (or digraph) is a pair $(N, D)$, where $N \subset \mathbb{N}$ is a finite set of nodes and $D \subset N \times N$ is a binary relation which elements $(i, j) \in D$ are called $\operatorname{arcs}$ on $N$. We only consider digraphs $(N, D)$ that are irreflexive, i.e., $(i, i) \notin D$ for every $i \in N$. The collection of all digraphs is denoted by $\mathcal{D}$. For $i \in N$ and $(N, D) \in \mathcal{D}$, we define

$$
S_{(N, D)}(i)=\{j \in N \mid(i, j) \in D\}
$$

being the set of successors of node $i \in N$ in digraph $(N, D)$, and

$$
P_{(N, D)}(i)=\{j \in N \mid(j, i) \in D\}
$$

being the set of predecessors of $i$ in $(N, D)$. The outdegree out $t_{i}(N, D)$ and indegree $i n_{i}(N, D)$ of node $i$ in $(N, D)$ are the cardinalities of $S_{(N, D)}(i)$ and $P_{(N, D)}(i)$, respectively, i.e.,

$$
\operatorname{out}_{i}(N, D)=\# S_{(N, D)}(i) \text { and } i n_{i}(N, D)=\# P_{(N, D)}(i) .
$$

A preorder on $N$ is a binary relation $\mathcal{R} \subset N \times N$ that is reflexive (i.e., $(i, i) \in \mathcal{R}$ for all $i \in N$ ) and transitive (i.e., if $(i, j) \in \mathcal{R}$ and $(j, h) \in \mathcal{R}$, then $(i, h) \in \mathcal{R}$ for every $i, j, h \in N)$. A preorder $\mathcal{R}$ on $N$ is complete if $(i, j) \in \mathcal{R}$ or $(j, i) \in \mathcal{R}$ for every pair $i, j \in N, i \neq j$. We use the standard notation: $i \succeq j$ (meaning that $i$ is ranked at least as high as $j$ ) if and only if $(i, j) \in \mathcal{R} ; i \succ j$ (meaning that $i$ is ranked higher than $j$ ) if and only if $[i \succeq j$ and not $j \succeq i]$; and $i \sim j$ (meaning that $i$ and $j$ are ranked equally) if and only if $[i \succeq j$ as well as $j \succeq i]$. The collection of all complete preorders on $N$ is denoted by $\mathcal{W}$.

A ranking method is a mapping $R: \mathcal{D} \rightarrow \mathcal{W}$ which assigns to every digraph $(N, D) \in \mathcal{D}$ on $N$ a complete preorder $R(N, D) \in \mathcal{W}$. We use the notation $i \succeq_{(N, D)} j$ if and only if $(i, j) \in R(N, D)$.

One of the most famous ranking methods for digraphs is the ranking by Copeland score, where the Copeland score $\operatorname{cop}_{i}(N, D)$ of node $i \in N$ in digraph $(N, D)$ is given by

$$
\operatorname{cop}_{i}(N, D)=\operatorname{out}_{i}(N, D)-i n_{i}(N, D)
$$

Then, the ranking by Copeland score is the ranking method given by

$$
i \succeq_{(N, D)}^{c o p} j \Leftrightarrow \operatorname{cop}_{i}(N, D) \geq \operatorname{cop}_{j}(N, D) \text { for all } i, j \in N \text {. }
$$

In the next section, we introduce and characterize a different ranking method based on the outdegree and indegree. 


\section{Axiomatization of the ranking by degree ratio}

Whereas the Copeland score assigns to every node in a digraph its outdegree minus its indegree, the degree ratio assigns to every node in a digraph its outdegree divided by its indegree. To avoid dividing by zero, we somehow assume the digraph to be reflexive, and therefore add 1 to both the out- as well as indegree of every node. ${ }^{2}$ Notice that doing the same for the Copeland score does not change the scores, but it has an effect for the degree ratio.

Definition 1 The degree ratio of node $i \in N$ in digraph $(N, D)$ is given by

$$
r_{i}(N, D)=\frac{\text { out }_{i}(N, D)+1}{\operatorname{in}_{i}(N, D)+1} .
$$

Definition 2 The ranking by degree ratio is the ranking method given by

$$
i \succeq_{(N, D)}^{d r} j \Leftrightarrow r_{i}(N, D) \geq r_{j}(N, D) \text { for all } i, j \in N
$$

The ranking by degree ratio is different from the ranking by Copeland score, and it is easy to construct examples such that for two nodes $i, j \in N, \operatorname{cop}_{i}(N, D)<\operatorname{cop}_{j}(N, D)$ while $r_{i}(N, D)>r_{j}(N, D)$. This happens, e.g., when

$$
i n_{i}(N, D)=0 \quad \text { and } \quad \frac{\text { out }_{j}(N, D)-i n_{j}(N, D)}{i n_{j}(N, D)+1}<\operatorname{out}_{i}(N, D)<\operatorname{out}_{j}(N, D)-i n_{j}(N, D) .
$$

Example 1 Consider the digraph $(N, D)$ with $N=\{1,2, \ldots, 9\}$ and $D=\{(1,2)\} \cup$ $\{(i, 5) \mid i=3,4\} \cup\{(5, i) \mid i=6,7,8,9\}$ as presented in Figure 1.

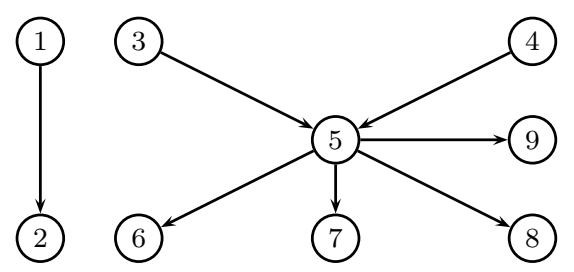

Fig. 1. Example 1

Table 1 shows the out- and indegree, the Copeland score and the degree ratio for every node. The last row shows the modified degree ratio $\bar{r}$ which is discussed in Section 5.

Note that $\operatorname{cop}_{1}(N, D)=1<2=\operatorname{cop}_{5}(N, D)$ but $r_{1}(N, D)=2>\frac{5}{3}=r_{5}(N, D)$. Moreover, $\frac{\text { out }_{5}(N, D)-i n_{5}(N, D)}{\operatorname{in}_{5}(N, D)+1}=\frac{2}{3}<1=$ out $_{1}(N, D)<2=\operatorname{out}_{5}(N, D)-i n_{5}(N, D)$.

Another property of the degree ratio concerns draws, i.e., two arcs between the same nodes oriented in opposite direction. A draw is not neutral in the degree ratio, contrary to the Copeland score which is indifferent to an additional draw. More precisely, when an agent loses more frequently than gains, he benefits from an additional draw. Moreover, if

\footnotetext{
${ }^{2}$ We could as well assume the digraph to be reflexive, and adapt various notations in the paper.
} 


\begin{tabular}{|c|c|c|c|}
\hline$i$ & $1,3,4$ & $2,6,7,8,9$ & 5 \\
\hline out $_{i}(N, D)$ & 1 & 0 & 4 \\
\hline in $_{i}(N, D)$ & 0 & 1 & 2 \\
\hline $\operatorname{cop}_{i}(N, D)$ & 1 & -1 & 2 \\
\hline$r_{i}(N, D)$ & 2 & $1 / 2$ & $5 / 3$ \\
\hline $\bar{r}_{i}(N, D)$ & $\infty$ & 0 & 2 \\
\hline
\end{tabular}

Table 1. Example 1

an agent gains more frequently than loses, an additional draw is disadvantageous to him, i.e., one can say that being a winner requires keeping the good image. Formally, we have the following fact.

Fact 1 Let $(N, D),\left(N, D^{\prime}\right) \in \mathcal{D}, i \in N$ and $x \in \mathbb{N}_{+}$be such that $i n_{i}\left(N, D^{\prime}\right)=i n_{i}(N, D)+$ $x$ and $\operatorname{out}_{i}\left(N, D^{\prime}\right)=\operatorname{out}_{i}(N, D)+x$. Then $\operatorname{cop}_{i}\left(N, D^{\prime}\right)=\operatorname{cop}_{i}(N, D)$ and

$$
r_{i}\left(N, D^{\prime}\right)>r_{i}(N, D) \quad \text { if and only if } \quad i n_{i}(N, D)>\text { out }_{i}(N, D) .
$$

Proof Indeed, we have

$$
\begin{gathered}
r_{i}\left(N, D^{\prime}\right)=\frac{\text { out }_{i}\left(N, D^{\prime}\right)+1}{i n_{i}\left(N, D^{\prime}\right)+1}=\frac{\text { out }_{i}(N, D)+x+1}{i n_{i}(N, D)+x+1}>\frac{\text { out }_{i}(N, D)+1}{i n_{i}(N, D)+1}=r_{i}(N, D) \\
\left.\left.\Leftrightarrow \operatorname{out}_{i}(N, D)+x+1\right)\left(\operatorname{in}_{i}(N, D)+1\right)>\operatorname{out}_{i}(N, D)+1\right)\left(\operatorname{in}_{i}(N, D)+x+1\right) \\
\Leftrightarrow \operatorname{in}_{i}(N, D)>\operatorname{out}_{i}(N, D)
\end{gathered}
$$

Hence, with the out- and indegree being interpreted as the number of gains and losses, respectively, the degree ratio can be used, for instance, for ranking candidates in situations when losing is seen more negatively than winning is seen positively. ${ }^{3}$

In what follows, we deliver an axiomatic characterization of the degree ratio. The first axiom is the 'standard' anonymity axiom stating that relabeling the nodes implies a corresponding relabeling in the ranking. For digraph $(N, D) \in \mathcal{D}$ and permutation $\pi: N \rightarrow N$, the permuted digraph is $(N, \pi D)$ with $\pi D=\bigcup_{(i, j) \in D}(\pi(i), \pi(j))$.

Axiom 1 (Anonymity) For every $(N, D) \in \mathcal{D}$, it holds that $i \succeq_{(N, D)} j \Leftrightarrow \pi(i) \succeq_{(N, \pi D)}$ $\pi(j)$.

Positive responsiveness implies that, when two nodes are equally ranked, and one of the nodes gets one more successor, then this node becomes strictly higher ranked than the other node.

Axiom 2 (Positive responsiveness) Let $(N, D),\left(N, D^{\prime}\right) \in \mathcal{D}$ and $i \in N$ be such that

$$
D^{\prime}=D \cup\{(i, g)\} \text { for some } g \in N \backslash\{i\} \text {. }
$$

Then $i \succeq_{(N, D)} j \Rightarrow i \succ_{\left(N, D^{\prime}\right)} j$ for all $j \in N \backslash\{i\}$.

\footnotetext{
${ }^{3}$ Although we do not consider utility in this paper, this is similar to risk attitudes in prospect theory, see Kahneman and Tversky (1979).
} 
The next axiom is independence of irrelevant arcs which implies that for the pairwise comparison between two nodes, we only need to consider the arcs that involve these two nodes.

Axiom 3 (Independence of irrelevant arcs) Let $(N, D),\left(N, D^{\prime}\right) \in \mathcal{D}$ and $i, j \in N$ be such that $S_{(N, D)}(i)=S_{\left(N, D^{\prime}\right)}(i), P_{(N, D)}(i)=P_{\left(N, D^{\prime}\right)}(i), S_{(N, D)}(j)=S_{\left(N, D^{\prime}\right)}(j)$ and $P_{(N, D)}(j)=P_{\left(N, D^{\prime}\right)}(j)$. Then $i \succeq_{(N, D)} j \Leftrightarrow i \succeq_{\left(N, D^{\prime}\right)} j$.

This axiom is weaker than independence of irrelevant arcs as used in van den Brink and Gilles (2003), generalizing an axiom of Rubinstein (1980), to axiomatize the ranking by outdegree, which requires that the ranking between two nodes does not change as long as their sets of successors do not change. An alternative weakening of van den Brink and Gilles (2003)'s independence of irrelevant arcs is used by Bouyssou and Marchant (2018). They consider an arc irrelevant for the pairwise comparison between nodes $i$ and $j$, if none of these two nodes is predecessor on this arc, nor is any of their successors the successor on the arc. So, whereas we take into account the relations with the predecessors of $i$ and $j$, Bouyssou and Marchant (2018) take account of the relations of the successors of $i$ and $j$ with their predecessors. These two weakenings of van den Brink and Gilles (2003)'s independence of irrelevant arcs are not logically related.

All axioms above are also satisfied by the ranking by Copeland score. Anonymity, positive responsiveness and negative responsiveness ${ }^{4}$ are used by Bouyssou (1992) to axiomatize the ranking by Copeland score. Instead of independence of irrelevant arcs, he uses independence of 2- or 3-cycles, meaning that deleting a cycle of length 2 or 3 does not change the ranking.

Next, we introduce a new type of property that is not satisfied by the ranking by Copeland score. This concerns the entrants of new nodes. Suppose that node $i$ and all its direct relatives (i.e. all its successors and predecessors) get a 'sibling'. Assume that agent $i$ is symmetrically related to its own sibling, i.e. is its predecessor as well as successor. If agent $i$ is related to the siblings of its relatives in exactly the same way as to its original relatives, (i.e., $i$ becomes successor of the siblings of all its predecessors, and becomes predecessor of the siblings of all its successors), then the ranking between node $i$ and any other original node $j$ who is not related to node $i$, should not change.

Axiom 4 (sibling neutrality 1) Let $(N, D) \in \mathcal{D}$ and $i \in N$, and let $S^{\prime}, P^{\prime} \subset \mathbb{N} \backslash N$ be such that $\# S^{\prime}=\# S_{(N, D)}(i)$, \# $P^{\prime}=\# P_{(N, D)}(i)$ and $\#\left(S^{\prime} \cap P^{\prime}\right)=\#\left(S_{(N, D)}(i) \cap P_{(N, D)}(i)\right)$. Further, let $\left(N^{\prime}, D^{\prime}\right)$ be given by $N^{\prime}=N \cup S^{\prime} \cup P^{\prime} \cup\left\{i^{\prime}\right\}, i^{\prime} \notin N \cup S^{\prime} \cup P^{\prime}$, and

$$
D^{\prime}=D \cup\left\{(h, i) \mid h \in P^{\prime}\right\} \cup\left\{(i, h) \mid h \in S^{\prime}\right\} \cup\left\{\left(i, i^{\prime}\right),\left(i^{\prime}, i\right)\right\}
$$

Then $i \succeq_{(N, D)} j \Leftrightarrow i \succeq_{\left(N^{\prime}, D^{\prime}\right)} j$ for all $j \in N \backslash\left(P_{(N, D)}(i) \cup S_{(N, D)}(i)\right)$.

Bouyssou and Marchant (2018) consider a different axiom that allows new nodes to enter that are in some sense related to the existing nodes. First, they introduce node addition, which states that "the addition of nodes that are not linked to any other node

\footnotetext{
${ }^{4}$ We do not need the axiom of negative responsiveness for characterizing the ranking by degree ratio, but in our framework this axiom would be defined as follows: Let $(N, D),\left(N, D^{\prime}\right) \in \mathcal{D}$ and $i \in N$ be such that $D^{\prime}=D \cup\{(g, i)\}$ for some $g \in N \backslash\{i\}$. Then $j \succeq_{(N, D)} i \Rightarrow j \succ_{\left(N, D^{\prime}\right)} i$ for all $j \in N \backslash\{i\}$.
} 


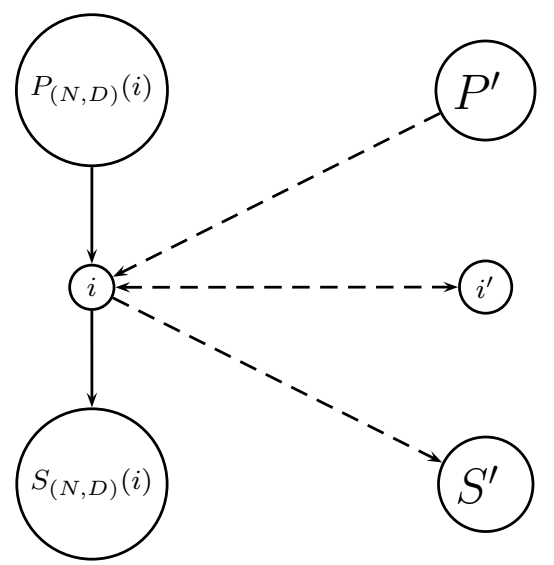

Fig. 2. Axiom 4 - entrance of a sibling of $i$ if $P_{(N, D)}(i) \cap S_{(N, D)}(i)=\emptyset$, the dashed arrows represent the added arcs.

has no influence on the ranking". Obviously, this axiom is also satisfied by the ranking by degree ratio. Second, they introduce independence of local density, stating that "increasing the number of successors of node $i$ and simultaneously increasing their number of predecessors, in the same proportion, does not improve or worsen the position of node $i$ ". Together with node addition this is a different way of adding new nodes that are related to $i$ in a special way, but is logically not related to sibling neutrality 1 .

Remark 1 In sibling neutrality 1, we allowed symmetric arcs between the node that gets a sibling and its successors/predecessors, i.e. $S_{(N, D)}(i) \cap P_{(N, D)}(i)$ might be nonempty. The uniqueness result presented below in Theorem 1 also holds if we weaken the sibling neutrality by not allowing symmetric arcs, i.e., if we only require the statement if $S_{(N, D)}(i) \cap P_{(N, D)}(i)=\emptyset$ for all $i \in N$, as was the case in the illustration in Figure 2.

Remark 2 Also, we required the ranking not to change between $i$ and any original node $j$ that is not related to $i$. This is sufficient in the characterization, but the ranking by degree ratio satisfies the stronger axiom where we require the ranking between $i$ and any other node in $N$ not to change.

The final axiom states that putting only one new node between a successor and a predecessor, without any further relations, does not change the ranking. This axiom is also satisfied by the ranking by Copeland score.

Axiom 5 (Intermediary property) Let $(N, D) \in \mathcal{D}, z \notin N$ and $i, j \in N$ be such that $(i, j) \in D$. Let $\left(N^{\prime}, D^{\prime}\right)$ be given by $N^{\prime}=N \cup\{z\}$ and

$$
D^{\prime}=(D \backslash\{(i, j)\}) \cup\{(i, z),(z, j)\} .
$$

Then $h \succeq_{(N, D)} g \Leftrightarrow h \succeq_{\left(N^{\prime}, D^{\prime}\right)} g$ for all $h, g \in N$.

The above axioms characterize the ranking by degree ratio.

Theorem 1 The ranking by degree ratio is the unique ranking method satisfying anonymity, positive responsiveness, independence of irrelevant arcs, sibling neutrality 1, and the intermediary property.

The proof can be found in the appendix. 


\section{A comparable axiomatization of the ranking by Copeland score}

From the axioms of Theorem 1, the ranking by Copeland score satisfies all except the sibling neutrality. However, it does satisfy an alternative sibling neutrality where, instead of putting all arcs as described in Axiom 4, we only link $i$ with its own copy $i^{\prime}$ by putting the arcs between $i$ and its copy $i^{\prime}$ in both directions. So, we only consider sibling $i^{\prime}$ as new entrant, who is symmetrically related to $i$. This entrance of a sibling is illustrated in Figure 3. (For transparency, the figure again only considers cases where there are no nodes that are successors as well as predecessors of node $i$ ).

Axiom 6 (sibling neutrality 2) Let $(N, D) \in \mathcal{D}$ and $i \in N$. Let $\left(N^{\prime}, D^{\prime}\right)$ be given by $N^{\prime}=N \cup\left\{i^{\prime}\right\}, i^{\prime} \notin N$, and $D^{\prime}=D \cup\left\{\left(i, i^{\prime}\right),\left(i^{\prime}, i\right)\right\}$. Then $i \succeq_{(N, D)} j \Leftrightarrow i \succeq_{\left(N^{\prime}, D^{\prime}\right)} j$ for all $j \in N$.

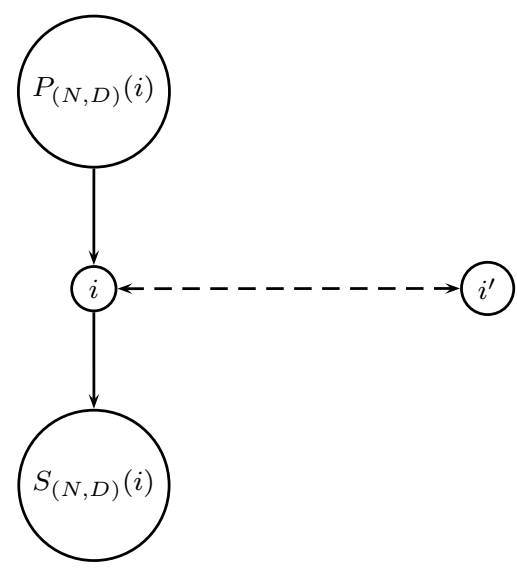

Fig. 3. Axiom 6 - entrance of a sibling of $i$ if $P_{(N, D)}(i) \cap S_{(N, D)}(i)=\emptyset$, the dashed arrows represent the added arcs.

Replacing sibling neutrality 1 in Theorem 1 by sibling neutrality 2, characterizes the ranking by Coplend score.

Theorem 2 The ranking by Copeland score is the unique ranking method satisfying anonymity, positive responsiveness, independence of irrelevant arcs, sibling neutrality 2 , and the intermediary property.

Also, the proof of this theorem can be found in the appendix.

Theorems 1 and 2 present comparable axiomatic characterizations of the rankings by degree ratio and Copeland score, respectively, that only differ in one axiom - the sibling neutrality. 


\section{A modification of the degree ratio choosing strong maximal nodes}

In Section 3, we defined the degree ratio as the ratio between the outdegree and indegree after making the digraph reflexive, avoiding dividing by a zero indegree. However, we can also simply use the outdegree divided by the indegree (without adding 1's) whenever the indegree is nonzero, and specify the ranking if there are nodes with zero indegree. An obvious way to do this is (i) to always rank strong maximal nodes, i.e., nodes with zero indegree ${ }^{5}$, above nodes with positive indegree, and (ii) if there are multiple strong maximal nodes, then to rank them by outdegree. This keeps the "spirit" of the degree ratio, but in some situations ranks nodes that were never losing above those with a positive indegree, despite the inverse ranking by the original degree ratio.

Definition 3 The modified degree ratio of node $i \in N$ in digraph $(N, D)$ is given by

$$
\bar{r}_{i}(N, D)=\left\{\begin{array}{cl}
\frac{\text { out }_{i}(N, D)}{i n_{i}(N, D)} & \text { if in } n_{i}(N, D)>0 \\
\infty & \text { if } i n_{i}(N, D)=0
\end{array}\right.
$$

Definition 4 The ranking by modified degree ratio is the ranking method given by

$$
i \succeq_{(N, D)}^{m d r} j \Leftrightarrow \Leftrightarrow\left\{\begin{array}{l}
\bar{r}_{i}(N, D) \geq \bar{r}_{j}(N, D) \text { and } i_{i}(N, D) \cdot i n_{j}(N, D)>0 \\
\text { or } \\
i n_{i}(N, D)=0 \text { and } i n_{j}(N, D)>0 \\
\text { or } \\
i n_{i}(N, D)=i n_{j}(N, D)=0 \text { and out }(N, D) \geq \text { out }_{j}(N, D) .
\end{array}\right.
$$

The ranking by modified degree ratio being different from the ranking by Copeland score can be seen from Example 1, where $\operatorname{cop}_{5}(N, D)=2>1=\operatorname{cop}_{1}(N, D)$, but according to the modified degree ratio, node 1 is ranked higher than node 5 since it has zero indegree.

As illustrated in the following example, the ranking by modified degree ratio is also different from the ranking by degree ratio.

Example 2 Consider $(N, D)$ with $N=\{1,2,3,4,5\}$ and $D=\{(3,2),(2,4),(2,5)\}$ as illustrated in Figure 4. Table 2 presents the degrees and the scores in question for every node.

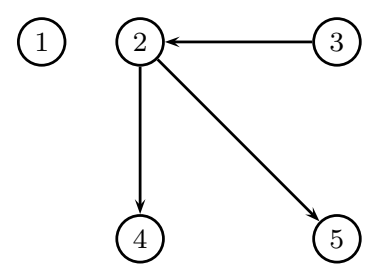

Fig. 4. Example 2

\footnotetext{
${ }^{5}$ A node is usually called maximal if it is not strictly defeated by any other node, i.e. if every predecessor is also a successor. We speak about a strong maximal node if it does not have any predecessor at all.
} 


\begin{tabular}{|c|c|c|c|c|}
\hline$i$ & 1 & 2 & 3 & 4,5 \\
\hline out $_{i}(N, D)$ & 0 & 2 & 1 & 0 \\
\hline$i n_{i}(N, D)$ & 0 & 1 & 0 & 1 \\
\hline $\operatorname{cop}_{i}(N, D)$ & 0 & 1 & 1 & -1 \\
\hline$r_{i}(N, D)$ & 1 & $3 / 2$ & 2 & $1 / 2$ \\
\hline $\bar{r}_{i}(N, D)$ & $\infty$ & 2 & $\infty$ & 0 \\
\hline
\end{tabular}

Table 2. Example 2

Then $r_{1}(N, D)=1<\frac{3}{2}=r_{2}(N, D)$, but according to the modified degree ratio, node 1 is ranked higher than node 2 since it has zero indegree. This example also shows that the ranking by Copeland score is different from the rankings by degree ratio and modified degree ratio: $\operatorname{cop}_{2}(N, D)=1=\operatorname{cop}_{3}(N, D)$ while $r_{2}(N, D)<r_{3}(N, D)$ and node 3 is also ranked higher according to the modified degree ratio. Note that $i n_{1}(N, D)=i n_{3}(N, D)=0$, but node 3 is ranked higher than node 1 according to the modified degree ratio, since out $_{3}(N, D)=1>0=$ out $_{1}(N, D)$.

The ranking by modified degree ratio can be different from the ranking by degree ratio due to strong maximal nodes, but not necessarily because of that. Moreover, if the degree and modified degree ratios give different ranking of two nodes with positive indegrees, then their ranking by Copeland score coincides with their ranking by degree ratio. From the definitions of the rankings by degree ratio and modified degree ratio, we get immediately the following fact.

Fact 2 Let $i n_{i}(N, D) \cdot i n_{j}(N, D)>0$. Then, for every $i, j \in N$,

(i) If $\left[\bar{r}_{i}(N, D)<\bar{r}_{j}(N, D)\right.$ and $\left.r_{i}(N, D)>r_{j}(N, D)\right]$ then $\operatorname{cop}_{i}(N, D)>\operatorname{cop}_{j}(N, D)$.

(ii) If $\left[\bar{r}_{i}(N, D)>\bar{r}_{j}(N, D)\right.$ and $\left.r_{i}(N, D)<r_{j}(N, D)\right]$ then $\operatorname{cop}_{i}(N, D)<\operatorname{cop}_{j}(N, D)$.

Proof (i) Let $i n_{i}(N, D) \cdot i n_{j}(N, D)>0, \bar{r}_{i}(N, D)<\bar{r}_{j}(N, D)$ and $r_{i}(N, D)>r_{j}(N, D)$ for some $i, j \in N$. This means that $\frac{\text { out }_{i}(N, D)}{i n_{i}(N, D)}<\frac{\text { out }_{j}(N, D)}{i n_{j}(N, D)}$ and $\frac{\text { out }_{i}(N, D)+1}{i n_{i}(N, D)+1}>\frac{\text { out }_{j}(N, D)+1}{i n_{j}(N, D)+1}$. Therefore, out $_{i}(N, D) \cdot i n_{j}(N, D)<$ out $_{j}(N, D) \cdot i n_{i}(N, D)$ and $\operatorname{out}_{i}(N, D) \cdot i n_{j}(N, D)+$ $\operatorname{out}_{i}(N, D)+i n_{j}(N, D)>\operatorname{out}_{j}(N, D) \cdot i n_{i}(N, D)+$ out $_{j}(N, D)+i n_{i}(N, D)$. This implies that $\operatorname{out}_{i}(N, D)+i n_{j}(N, D)>\operatorname{out}_{j}(N, D)+i n_{i}(N, D)$, and hence $\operatorname{cop}_{i}(N, D)>\operatorname{cop}_{j}(N, D)$.

(ii) It follows similarly to (i).

Example 3 Consider the digraph $(N, D)$ with $N=\{1,2, \ldots, 8\}$ and $D=\{(1,2),(5,8)\} \cup$ $\{(i, 5) \mid i=3,4,6,7\}$ as presented in Figure 5, with the corresponding Table 3.

Note that $r_{2}(N, D)=\frac{1}{2}>\frac{2}{5}=r_{5}(N, D)$, but $\bar{r}_{2}(N, D)=0<\frac{1}{4}=\bar{r}_{5}(N, D)$. Moreover, $\operatorname{cop}_{2}(N, D)=-1>-3=\operatorname{cop}_{5}(N, D)$, so the pairwise ranking between nodes 2 and 5 according to the Copeland score and degree ratio coincide.

The ranking by modified degree ratio can be axiomatized by similar axioms as before, except that we consider yet a different type of sibling neutrality, and add an axiom that explicitly requires to rank nodes with zero indegree above nodes with positive indegree.

sibling neutrality 2 used a subset of the arcs and nodes used in sibling neutrality 1. Putting the arcs and nodes that are used in sibling neutrality 1 , but not in sibling 


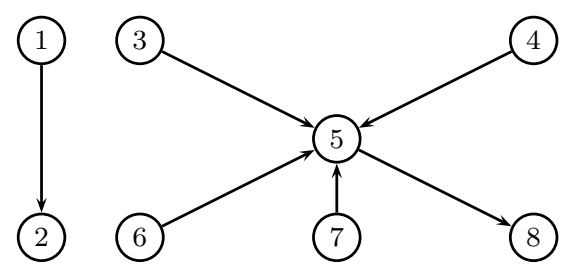

Fig. 5. Example 3

\begin{tabular}{|c|c|c|c|}
\hline$i$ & $1,3,4,6,7$ & 2,8 & 5 \\
\hline out $_{i}(N, D)$ & 1 & 0 & 1 \\
\hline in $_{i}(N, D)$ & 0 & 1 & 4 \\
\hline $\operatorname{cop}_{i}(N, D)$ & 1 & -1 & -3 \\
\hline$r_{i}(N, D)$ & 2 & $1 / 2$ & $2 / 5$ \\
\hline $\bar{r}_{i}(N, D)$ & $\infty$ & 0 & $1 / 4$ \\
\hline
\end{tabular}

Table 3. Example 3

neutrality 2 , we get another sibling neutrality that turns out to be satisfied by the ranking by modified degree ratio. In this sibling neutrality, new siblings of the predecessors and successors of agent $i$ enter, and these new siblings are related to node $i$ in the same way as their original nodes. This sibling neutrality is illustrated in Figure 6. (For transparency, the figure again only considers cases where there are no nodes that are successors as well as predecessors of node $i$ ).

Axiom 7 (sibling neutrality 3) Let $(N, D) \in \mathcal{D}$ and $i \in N$ with $P_{D}(i) \neq \emptyset$. Let

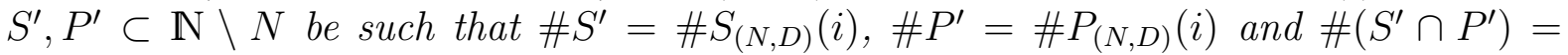
$\#\left(S_{(N, D)}(i) \cap P_{(N, D)}(i)\right)$. Further, let $\left(N^{\prime}, D^{\prime}\right)$ be given by $N^{\prime}=N \cup S^{\prime} \cup P^{\prime}$, and

$$
D^{\prime}=D \cup\left\{(h, i) \mid h \in P^{\prime}\right\} \cup\left\{(i, h) \mid h \in S^{\prime}\right\}
$$

Then $i \succeq_{(N, D)} j \Leftrightarrow i \succeq_{\left(N^{\prime}, D^{\prime}\right)} j$ for all $j \in N \backslash\left(P_{(N, D)}(i) \cup S_{(N, D)}(i)\right)$.

Additionally, we require that we refine the set of strong maximal nodes in the sense that, if a strong maximal node exists, then the node that is ranked highest is a strong maximal node. A node is called a strong maximal node if it does not have any predecessor. Let

$$
\operatorname{SMAX}(N, D)=\left\{i \in N \mid P_{(N, D)}(i)=\emptyset\right\}
$$

be the set of strong maximal nodes of $(N, D)$.

Axiom 8 (Maximal property) If $i \in \operatorname{SMAX}(N, D)$ and $j \in N \backslash \operatorname{SMAX}(N, D)$, then $i \succ_{(N, D)} j$.

Replacing sibling neutrality 1 in Theorem 1 by sibling neutrality 3, and adding the maximal property, characterizes the ranking by modified degree ratio. 


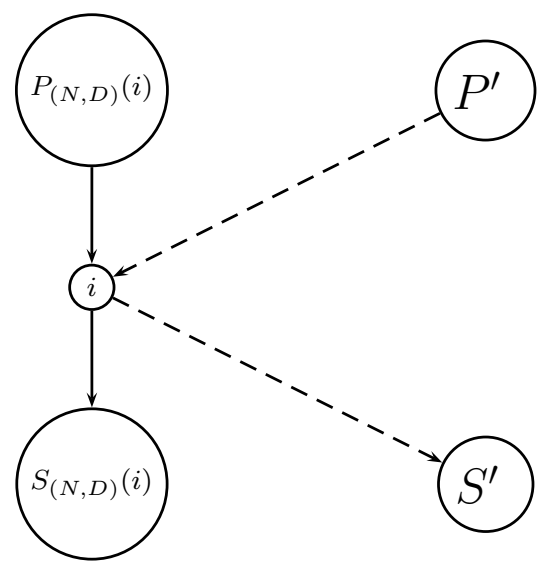

Fig. 6. Axiom 7 - entrance of a sibling of $i$ if $P_{(N, D)}(i) \cap S_{(N, D)}(i)=\emptyset$, the dashed arrows represent the added arcs.

Theorem 3 The ranking by modified degree ratio is the unique ranking method satisfying anonymity, positive responsiveness, independence of irrelevant arcs, sibling neutrality 3, the intermediary property, and the maximal property.

The proof can also be found in the appendix.

The set of added arcs between new and old nodes in sibling neutrality 2 and sibling neutrality 3, form a partition of the set of added arcs between new and old nodes in sibling neutrality 1 (assuming that the added sets $S^{\prime}$ and $P^{\prime}$ in sibling neutrality 1 and 3 are the same). Hence, applying the sibling neutrality where we add the arcs between new and old nodes that underlie the ranking by degree ratio, and leaving out those that underlie the ranking by Copeland score, gives the ranking by modified degree ratio.

\section{Conclusion}

This paper considers the ranking by degree ratio and ranking by modified degree ratio, as alternatives to the famous ranking by Copeland score. Similar as the ranking by Copeland score, these rankings are based on the out- and indegree, but instead of taking the difference between the out- and indegree, we divide the out- by indegree. In order to avoid dividing by a zero indegree, for the degree ratio we implicitly take the outand indegree of the reflexive digraph. For the modified degree ratio, we do not consider the reflexive digraph, but by definition assume that nodes with indegree zero are ranked higher than nodes with a positive indegree. The main results of the paper are axiomatic characterizations of the rankings by degree ratio and by modified degree ratio, as well as a comparable axiomatization of the ranking by Copeland score. A main difference between these three axiomatic characterizations is in the sibling neutrality that is applied. Therefore, the axiomatizations in this paper allow us to compare the three ranking methods by their sibling neutrality property.

A plan for future research is to consider a degree ratio for weighted directed networks where the links can have different weights. This allows to consider applications such as migration flows, financial flows, investment flows, etc., as mentioned in the introduction. 


\section{Appendix: Proofs}

\section{A1: Proof of Theorem 1}

We prove Theorem 1 in several steps.

Lemma 1. Let $(N, D) \in \mathcal{D}$ and $i, j \in N$ be such that $S_{(N, D)}(i) \cap P_{(N, D)}(i)=S_{(N, D)}(i) \cap$ $P_{(N, D)}(j)=S_{(N, D)}(j) \cap P_{(N, D)}(i)=S_{(N, D)}(j) \cap P_{(N, D)}(j)=\emptyset$, and $(i, j),(j, i) \notin D$. If ranking method $\succeq$ satisfies the axioms of Theorem 1 , then $\left[i \succeq j\right.$ if and only if $r_{i}(N, D) \geq$ $\left.r_{j}(N, D)\right]$.

\section{PROOF}

We distinguish the following cases:

\section{Case A}

Suppose that $r_{i}(N, D)=r_{j}(N, D)$. We will shortly denote $r_{i}(N, D)$ and $r_{j}(N, D)$ as $r_{i}$, respectively $r_{j}$.

First, we enter a sibling of node $i\left(i n_{j}(N, D)\right.$ times $)$, and $j\left(i n_{i}(N, D)\right.$ times $)$ as in the sibling property, in order to create symmetric positions for them.

Therefore, define $P_{i}^{\prime}, P_{j}^{\prime}, S_{i}^{\prime}, S_{j}^{\prime}, I, J \subset \mathbb{N} \backslash N$ such that each pair of these sets is disjoint, and

- \#I $=i n_{j}(N, D), \# J=i n_{i}(N, D)$;

- \#P $P_{i}^{\prime}=\# P_{j}^{\prime}=i n_{i}(N, D) \cdot i n_{j}(N, D)$;

- \# $S_{i}^{\prime}=\operatorname{out}_{i}(N, D) \cdot i n_{j}(N, D)$, and \# $S_{j}^{\prime}=\operatorname{out}_{j}(N, D) \cdot i n_{i}(N, D)$.

Further, let digraph $\left(N^{\prime}, D^{\prime}\right) \in \mathcal{D}$ be given by

$$
N^{\prime}=N \cup S_{i}^{\prime} \cup S_{j}^{\prime} \cup P_{i}^{\prime} \cup P_{j}^{\prime} \cup I \cup J
$$

and

$$
\begin{aligned}
D^{\prime}= & D \cup\left(\bigcup_{h \in S_{i}^{\prime}}\{(i, h)\}\right) \cup\left(\bigcup_{h \in P_{i}^{\prime}}\{(h, i)\}\right) \cup\left(\bigcup_{h \in I}\{(i, h),(h, i)\}\right) \cup \\
& \left(\bigcup_{h \in S_{j}^{\prime}}\{(j, h)\}\right) \cup\left(\bigcup_{h \in P_{j}^{\prime}}\{(h, j)\}\right) \cup\left(\bigcup_{h \in J}\{(j, h),(h, j)\}\right)
\end{aligned}
$$

This entrance of siblings of nodes $i$ and $j$ is illustrated in Figure 7, where the dashed arrows represent new added arcs, and the solid arrows represent already existing arcs in $D$.

To get rid of the asymmetry between nodes $i$ and $j$ with respect to the different sizes of the sets $I$, respectively $J$, we put nodes on the arcs between $i$ (respectively $j$ ) and the nodes in the set $I$ (respectively the set $J$ ) in both directions, which does not influence the ranking between nodes $i$ and $j$ by the intermediary property. This gives the following digraph $\left(N^{\prime \prime}, D^{\prime \prime}\right)$. Without loss of generality, let the nodes in $I$ be labeled as $I=\left\{i_{1}, \ldots, i_{s}\right\}$, and thus $\# I=i n_{j}(N, D)=s$. Similar, let the nodes in $J$ be labeled as $J=\left\{j_{1}, \ldots, j_{t}\right\}$ and thus $\# J=i n_{i}(N, D)=t$. Now, let $N^{\prime \prime}=N^{\prime} \cup I^{\prime} \cup J^{\prime}$ with $I^{\prime}, J^{\prime} \subset \mathbb{N} \backslash N^{\prime}, \# I^{\prime}=2 \# I$ 
and $\# J^{\prime}=2 \# J$, and suppose that the nodes in the two new sets are labeled as follows: $I^{\prime}=\left\{i_{11}^{\prime}, \ldots, i_{1 s}^{\prime}, i_{21}^{\prime}, \ldots, i_{2 s}^{\prime}\right\}$ and $J^{\prime}=\left\{j_{11}^{\prime}, \ldots, j_{1 t}^{\prime}, j_{21}^{\prime}, \ldots, j_{2 t}^{\prime}\right\}$. Further, let

$$
\begin{aligned}
D^{\prime \prime}=\left(D^{\prime}\right. & \left.\left.\backslash\left(\bigcup_{h \in I}\{(i, h),(h, i)\}\right) \cup\left(\bigcup_{h \in J}\{(j, h),(h, j)\}\right)\right)\right) \\
& \cup\left(\bigcup_{k=1}^{s}\left\{\left(i, i_{1 k}^{\prime}\right),\left(i_{1 k}^{\prime}, i_{k}\right),\left(i_{k}, i_{2 k}^{\prime}\right),\left(i_{2 k}^{\prime}, i\right)\right\}\right) \\
& \cup\left(\bigcup_{k=1}^{t}\left\{\left(j, j_{1 k}^{\prime}\right),\left(j_{1 k}^{\prime}, j_{k}\right),\left(j_{k}, j_{2 k}^{\prime}\right),\left(j_{2 k}^{\prime}, j\right)\right\}\right)
\end{aligned}
$$

being the digraph where we put intermediary nodes on all arcs between $i$, respectively $j$, and the nodes in the sets $I$, respectively $J$.

Next, let $\left(N^{\prime \prime}, D^{\prime \prime \prime}\right)$ be given by

$$
D^{\prime \prime \prime}=\left\{(h, g) \in D^{\prime \prime} \mid\{h, g\} \cap\{i, j\} \neq \emptyset\right\},
$$

where we deleted all 'irrelevant arcs' not containing $i$ or $j$.

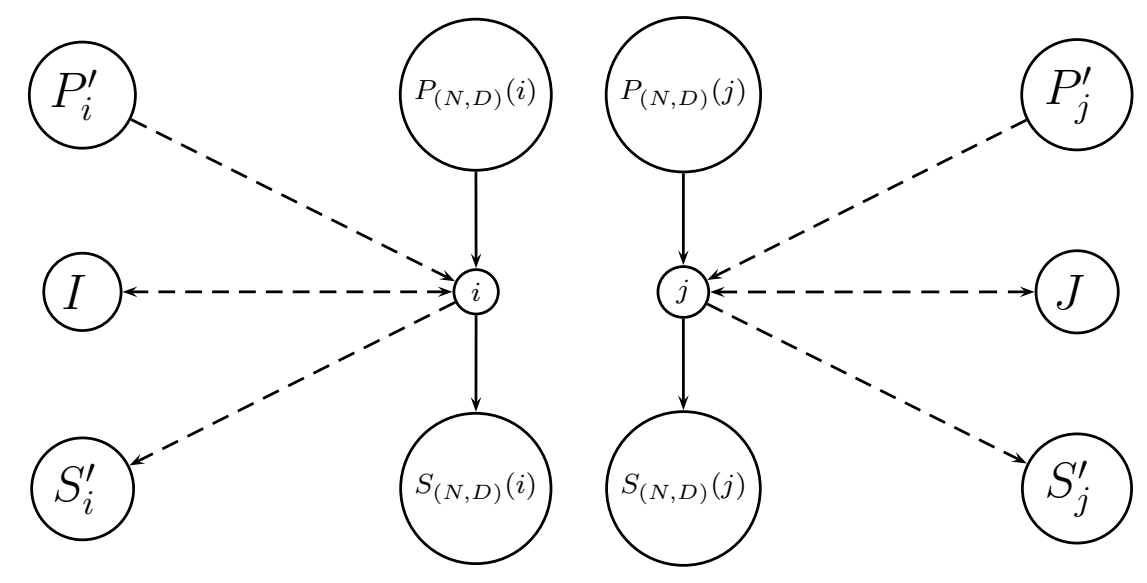

Fig. 7. Proof of Lemma 1 - Case A

Notice that

(i) $\# P_{\left(N^{\prime \prime}, D^{\prime \prime \prime}\right)}(i)=\# P_{i}^{\prime}+\# P_{i}(N, D)+\# I=i n_{i}(N, D) \cdot i n_{j}(N, D)+i n_{i}(N, D)+$ $i n_{j}(N, D)=\# P_{j}^{\prime}+\# J+\# P_{j}(N, D)=\# P_{\left(N^{\prime \prime}, D^{\prime \prime \prime}\right)}(j)$, and

(ii) $\# S_{\left(N^{\prime \prime}, D^{\prime \prime \prime}\right)}(i)=\# S_{i}^{\prime}+\# S_{i}(N, D)+\# I=\operatorname{out}_{i}(N, D) \cdot \operatorname{in}_{j}(N, D)+\operatorname{out}_{i}(N, D)+$ $\left.\operatorname{in}_{j}(N, D)=\operatorname{out}_{i}(N, D)+1\right)\left(\operatorname{in}_{j}(N, D)+1\right)-1=\left(\operatorname{out}_{j}(N, D)+1\right)\left(\operatorname{in}_{i}(N, D)+1\right)-1=$ $\operatorname{out}_{j}(N, D) \cdot i n_{i}(N, D)+\operatorname{out}_{j}(N, D)+i n_{i}(N, D)=\# S_{j}^{\prime}+\# S_{j}(N, D)+\# J=\# S_{\left(N^{\prime \prime}, D^{\prime \prime \prime}\right)}(j)$, where the fourth equality follows from the assumption that $r_{i}(N, D)=r_{j}(N, D)$.

Therefore, anonymity implies that $i \sim_{\left(N^{\prime \prime}, D^{\prime \prime \prime}\right)} j$. 
Independence of irrelevant arcs implies that the ranking between $i$ and $j$ is the same in $\left(N^{\prime \prime}, D^{\prime \prime}\right)$ as in $\left(N^{\prime \prime}, D^{\prime \prime \prime}\right)$, and thus $i \sim_{\left(N^{\prime \prime}, D^{\prime \prime}\right)} j$.

The intermediary property implies that the ranking between $i$ and $j$ is the same in $\left(N^{\prime}, D^{\prime}\right)$ as in $\left(N^{\prime \prime}, D^{\prime \prime}\right)$, and thus $i \sim_{\left(N^{\prime}, D^{\prime}\right)} j$.

Repeated application of sibling neutrality 1 implies that $i \sim_{(N, D)} j$.

\section{Case B}

Suppose without loss of generality that $r_{i}(N, D)>r_{j}(N, D)$.

We define similar sets $S_{i}^{\prime}, S_{j}^{\prime}, P_{i}^{\prime}, P_{j}^{\prime}, I$ and $J$ as in Case A, so define $P_{i}^{\prime}, P_{j}^{\prime}, S_{i}^{\prime}, S_{j}^{\prime}, I, J \subset$ $\mathbb{N} \backslash N$ such that each pair of these sets is disjoint, and

- \#I $=i n_{j}(N, D), \# J=i n_{i}(N, D)$;

- \#P $P_{i}^{\prime}=\# P_{j}^{\prime}=i n_{i}(N, D) \cdot i n_{j}(N, D)$;

- \# $S_{i}^{\prime}=\operatorname{out}_{i}(N, D) \cdot i n_{j}(N, D)$, and $\# S_{j}^{\prime}=\operatorname{out}_{j}(N, D) \cdot i n_{i}(N, D)$.

Define digraph $\left(N^{\prime}, D^{\prime}\right)$ as in Case A (see (2) and (3)).

Similar as in Case A, we put intermediary nodes on the arcs between nodes $i$ (respectively $j$ ) and the nodes in the set $I$ (respectively the set $J$ ), to obtain the digraph $\left(N^{\prime \prime}, D^{\prime \prime}\right)$ in the same way as in Case A, see (4).

Also, similar as in Case A, let $\left(N^{\prime \prime}, D^{\prime \prime \prime}\right)$ be the digraph where we deleted the irrelevant arcs', given by (5).

Similar as in Case A,

(i) $\# P_{\left(N^{\prime \prime}, D^{\prime \prime \prime}\right)}(i)=\# P_{i}^{\prime}+\# P_{i}(N, D)+\# I=i n_{i}(N, D) \cdot i n_{j}(N, D)+i n_{i}(N, D)+$ $i n_{j}(N, D)=\# P_{j}^{\prime}+\# J+\# P_{j}(N, D)=\# P_{\left(N^{\prime \prime}, D^{\prime \prime \prime}\right)}(j)$.

But now,

(ii) $\# S_{\left(N^{\prime \prime}, D^{\prime \prime \prime}\right)}(i)=\# S_{i}^{\prime}+\# S_{i}(N, D)+\# I=$ out $_{i}(N, D) \cdot$ in $_{j}(N, D)+$ out $_{i}(N, D)+$ $\left.\operatorname{in}_{j}(N, D)=\operatorname{out}_{i}(N, D)+1\right)\left(\operatorname{in}_{j}(N, D)+1\right)-1>\left(\operatorname{out}_{j}(N, D)+1\right)\left(\operatorname{in}_{i}(N, D)+1\right)-1=$ $\operatorname{out}_{j}(N, D) \cdot i n_{i}(N, D)+\operatorname{out}_{j}(N, D)+i n_{i}(N, D)=\# S_{j}^{\prime}+\# S_{j}(N, D)+\# J=\# S_{\left(N^{\prime \prime}, D^{\prime \prime \prime}\right)}(j)$, where the inequality follows from the assumption that $r_{i}(N, D)>r_{j}(N, D)$.

Let $D^{\prime \prime \prime \prime} \subset D^{\prime \prime \prime}$ with $\left[(h, g) \in D^{\prime \prime \prime} \backslash D^{\prime \prime \prime \prime} \Rightarrow h=i\right]$ and $\# S_{\left(N^{\prime \prime}, D^{\prime \prime \prime \prime}\right)}(i)=\# S_{\left(N^{\prime \prime}, D^{\prime \prime \prime \prime}\right)}(j)=$ $\# S_{\left(N^{\prime \prime}, D^{\prime \prime \prime}\right)}(j)$.

Since $\left(N^{\prime \prime}, D^{\prime \prime \prime \prime}\right)$ is as in Case A, we have that $i \sim_{\left(N^{\prime \prime}, D^{\prime \prime \prime \prime}\right)} j$.

Since $D^{\prime \prime \prime}$ is obtained from $D^{\prime \prime \prime \prime}$ by adding arcs with node $i$ as predecessor, repeated application of positive responsiveness implies that $i \succ_{\left(N^{\prime \prime}, D^{\prime \prime \prime}\right)} j$.

Independence of irrelevant arcs implies that the ranking between $i$ and $j$ is the same in $\left(N^{\prime \prime}, D^{\prime \prime}\right)$ as in $\left(N^{\prime \prime}, D^{\prime \prime \prime}\right)$, and thus $i \succ_{\left(N^{\prime \prime}, D^{\prime \prime}\right)} j$.

The intermediary property implies that the ranking between $i$ and $j$ is the same in $\left(N^{\prime}, D^{\prime}\right)$ as in $\left(N^{\prime \prime}, D^{\prime \prime}\right)$, and thus $i \succ_{\left(N^{\prime}, D^{\prime}\right)} j$.

Repeated application of sibling neutrality 1 implies that $i \succeq_{(N, D)} j$.

In a similar way, it can be shown that $j \nsucceq_{(N, D)} i$, and thus $i \succ_{(N, D)} j$.

\section{Proof of TheOREM 1}


To prove uniqueness, given Lemma 1 , we still have to consider the case where not all conditions $S_{(N, D)}(i) \cap P_{(N, D)}(i)=S_{(N, D)}(i) \cap P_{(N, D)}(j)=S_{(N, D)}(j) \cap P_{(N, D)}(i)=S_{(N, D)}(j) \cap$ $P_{(N, D)}(j)=\emptyset$ and $(i, j),(j, i) \notin D$ are satisfied. This is dealt with by the intermediary property.

First, if $(i, j) \in D$ then define $\left(N^{\prime}, D^{\prime}\right)$ by $N^{\prime}=N \cup\{z\}$ for some $z \notin N$, and $D^{\prime}=$ $(D \backslash\{(i, j)\}) \cup\{(i, z),(z, j)\}$. Similarly if $(j, i) \in D$. If $(i, j),(j, i) \notin D$, then simply take $\left(N^{\prime}, D^{\prime}\right)=(N, D)$.

Next, define

$$
\begin{gathered}
H=\left(\bigcup_{h \in S_{(N, D)}(i) \cap P_{(N, D)}(i)}\{(i, h)\}\right) \cup\left(\bigcup_{h \in S_{(N, D)}(i) \cap P_{(N, D)}(j)}\{(i, h)\}\right) \cup \\
\left(\bigcup_{h \in P_{(N, D)}(i) \cap S_{(N, D)}(j)}\{(j, h)\}\right) \cup\left(\bigcup_{h \in S_{(N, D)}(j) \cap P_{(N, D)}(j)}\{(j, h)\}\right),
\end{gathered}
$$

being the outgoing arcs (from $i$ or $j$ ) of the symmetric part involving nodes $i$ and $j$, and nodes that are directly related to both $i$ and $j$.

Then, define $\left(N^{\prime \prime}, D^{\prime \prime}\right)$ such that

$$
N^{\prime} \subset N^{\prime \prime} \text { with } \# N^{\prime \prime}=\# N^{\prime}+\# H
$$

and, labeling the nodes in $N^{\prime \prime} \backslash N^{\prime}$ as $N^{\prime \prime} \backslash N^{\prime}=\left\{z_{(h, g)} \mid(h, g) \in H\right\}$,

$$
D^{\prime \prime}=\left(D^{\prime} \backslash H\right) \cup\left\{\bigcup_{(h, g) \in H}\left\{\left(h, z_{(h, g)}\right),\left(z_{(h, g)}, g\right)\right\}\right\} .
$$

Thus, $i n_{z}\left(N^{\prime \prime}, D^{\prime \prime}\right)=\operatorname{out}_{z}\left(N^{\prime \prime}, D^{\prime \prime}\right)=1$ for all $z \in N^{\prime \prime} \backslash N^{\prime}$.

Since $\left(N^{\prime \prime}, D^{\prime \prime}\right)$ is as in Lemma 1 , we have $i \succeq_{\left(N^{\prime \prime}, D^{\prime \prime}\right)} j$ if and only if $i \succeq_{\left(N^{\prime \prime}, D^{\prime \prime}\right)}^{d r} j$.

The intermediary property then implies that $i \succeq_{(N, D)} j$ if and only if $i \succeq_{(N, D)}^{d r} j$.

We are left to show that the ranking by degree ratio satisfies the axioms.

$\succeq^{d r}$ satisfying anonymity is obvious.

$\succeq^{d r}$ satisfying positive responsiveness follows since the degree ratio of a node is increasing if its outdegree increases, which has no positive effect for the other nodes.

$\succeq^{d r}$ satisfying independence of irrelevant arcs follows since the degree ratio of a node only depends on the arcs with its successors and predecessors.

$\succeq^{d r}$ satisfying the sibling property follows since $r_{i}\left(N^{\prime}, D^{\prime}\right)=\frac{o u t_{i}\left(N^{\prime}, D^{\prime}\right)+1}{i n_{i}\left(N^{\prime}, D^{\prime}\right)+1}=\frac{2 o u t_{i}(N, D)+1+1}{2 i n_{i}(N, D)+1+1}=$ $\frac{2\left(\text { out }_{i}(N, D)+1\right)}{2\left(i_{i}(N, D)+1\right)}=\frac{\text { out }_{i}(N, D)+1}{i n_{i}(N, D)+1}=r_{i}(N, D)$, and $r_{j}\left(N^{\prime}, D^{\prime}\right)=r_{j}(N, D)$ for all $j \in N \backslash\{i\}$, where $\left(N^{\prime}, D^{\prime}\right)$ is as given in the axiom.

$\succeq^{d r}$ satisfying the intermediary property follows since $r_{j}\left(N^{\prime}, D^{\prime}\right)=r_{j}(N, D)$ for all $j \in N$ with $\left(N^{\prime}, D^{\prime}\right)$ as given in the axiom. 


\section{A2: Proof of Theorem 2}

We prove Theorem 2 in similar steps as the proof of Theorem 1.

Lemma 2. Let $(N, D) \in \mathcal{D}$ and $i, j \in N$ be such that $S_{(N, D)}(i) \cap P_{(N, D)}(i)=S_{(N, D)}(i) \cap$ $P_{(N, D)}(j)=S_{(N, D)}(j) \cap P_{(N, D)}(i)=S_{(N, D)}(j) \cap P_{(N, D)}(j)=\emptyset$, and $(i, j),(j, i) \notin D$. If ranking method $\succeq$ satisfies the axioms of Theorem 2 , then $[i \succeq j$ if and only if $\left.\operatorname{cop}_{i}(N, D) \geq \operatorname{cop}_{j}(N, D)\right]$.

\section{Proof}

We distinguish similar two cases as in the proof of Theorem 1.

\section{Case A}

Suppose that $\operatorname{cop}_{i}(N, D)=\operatorname{cop}_{j}(N, D)$. Suppose without loss of generality that out $_{i}(N, D) \geq$ out $_{j}(N, D)$ (and thus $\left.i n_{i}(N, D) \geq i n_{j}(N, D)\right)$.

First, we enter a sibling of node $j$ several times as in sibling neutrality 2 , in order to create symmetric positions for nodes $i$ and $j$. (Notice that in the proof of Theorem 1 we had to enter siblings of both nodes $i$ and $j$.) Therefore, for $J \subset \mathbb{N} \backslash N$ such that $\# J=$ out $_{i}(N, D)-$ out $_{j}(N, D)$, define digraph $\left(N^{\prime}, D^{\prime}\right) \in \mathcal{D}$ by

$$
N^{\prime}=N \cup J
$$

and

$$
D^{\prime}=D \cup\left(\bigcup_{h \in J}\{(h, j),(j, h)\}\right) .
$$

The entrance of a sibling of node $j$ is illustrated in Figure 8, where the dashed arrows represent new added arcs, and the solid arrows represent already existing arcs.

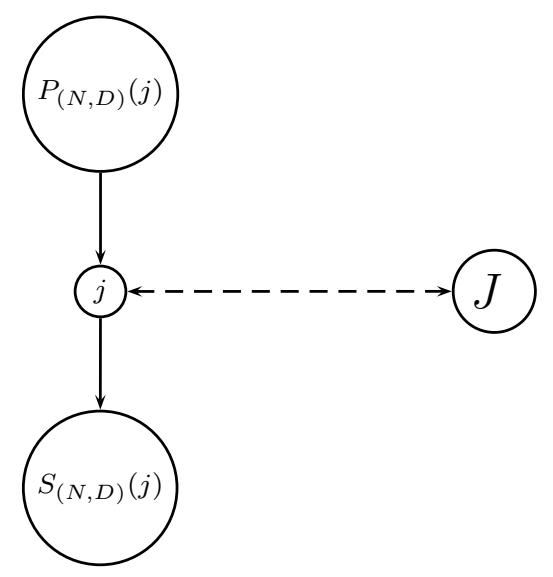

Fig. 8. Proof of Lemma 2 - Case A

To get rid of the asymmetry between nodes $i$ and $j$ with respect to the set $J$, we add intermediary nodes between $j$ and the nodes in the set $J$, which does not influence the ranking between nodes $i$ and $j$ by the intermediary property. This is done similar as 
in the proof of Theorem 1 , but now only considering $j$ and the set $J$. This gives the following digraph $\left(N^{\prime \prime}, D^{\prime \prime}\right)$. Without loss of generality, let the nodes in $J$ be labeled as $J=\left\{j_{1}, \ldots, j_{t}\right\}$ and thus $\# J=$ out $_{i}(N, D)-$ out $_{j}(N, D)=t$. Now, let $N^{\prime \prime}=N^{\prime} \cup J^{\prime}$ with $J^{\prime} \subset \mathbb{N} \backslash N^{\prime}$ such that $\# J^{\prime}=2 \# J$, and suppose that the nodes in the new set are labeled as follows: $J^{\prime}=\left\{j_{11}^{\prime}, \ldots, j_{1 t}^{\prime}, j_{21}^{\prime}, \ldots, j_{2 t}^{\prime}\right\}$. Further, let

$$
\begin{gathered}
D^{\prime \prime}=\left(D^{\prime} \backslash\left(\bigcup_{h \in J}\{(j, h),(h, j)\}\right)\right) \\
\cup\left(\bigcup_{k=1}^{t}\left\{\left(j, j_{1 k}^{\prime}\right),\left(j_{1 k}^{\prime}, j_{k}\right),\left(j_{k}, j_{2 k}^{\prime}\right),\left(j_{2 k}^{\prime}, j\right)\right\}\right)
\end{gathered}
$$

being the digraph where we put intermediary nodes on all arcs between $j$ and the nodes in the set $J$.

Similar as in the proof of Theorem 1 , let $\left(N^{\prime \prime}, D^{\prime \prime \prime}\right)$ be given by

$$
D^{\prime \prime \prime}=\left\{(h, g) \in D^{\prime \prime} \mid\{h, g\} \cap\{i, j\} \neq \emptyset\right\},
$$

where we deleted all 'irrelevant arcs' not containing $i$ or $j$.

Notice that $\# S_{\left(N^{\prime \prime}, D^{\prime \prime \prime}\right)}(j)=\# S_{(N, D)}(j)+\# J=\operatorname{out}_{j}(N, D)+\operatorname{out}_{i}(N, D)-$ out $_{j}(N, D)=$ $\operatorname{out}_{i}(N, D)=\# S_{\left(N^{\prime \prime}, D^{\prime \prime \prime}\right)}(i)$, and thus with $\operatorname{cop}_{i}(N, D)=\operatorname{cop}_{j}(N, D)$ it follows that $\# P_{\left(N^{\prime \prime}, D^{\prime \prime \prime}\right)}(j)=\# P_{(N, D)}(j)+\# J=i n_{j}(N, D)+\operatorname{out}_{i}(N, D)-\operatorname{out}_{j}(N, D)=i n_{i}(N, D)=$ $\# P_{\left(N^{\prime \prime}, D^{\prime \prime \prime}\right)}(i)$.

Therefore, anonymity implies that $i \sim_{\left(N^{\prime \prime}, D^{\prime \prime \prime}\right)} j$.

Independence of irrelevant arcs implies that the ranking between $i$ and $j$ is the same in $\left(N^{\prime \prime}, D^{\prime \prime}\right)$ as in $\left(N^{\prime \prime}, D^{\prime \prime \prime}\right)$, and thus $i \sim\left(N^{\prime \prime}, D^{\prime \prime}\right) j$.

The intermediary property implies that the ranking between $i$ and $j$ is the same in $\left(N^{\prime}, D^{\prime}\right)$ as in $\left(N^{\prime \prime}, D^{\prime \prime}\right)$, and thus $i \sim_{\left(N^{\prime}, D^{\prime}\right)} j$.

Similar as in the proof of Theorem 1 , repeated application of sibling neutrality 2 implies that $i \sim{ }_{(N, D)} j$.

\section{Case B}

Suppose without loss of generality that $\operatorname{cop}_{i}(N, D)>\operatorname{cop}_{j}(N, D)$.

If $i n_{i}(N, D)<i n_{j}(N, D)$ then take $I \subset \mathbb{N} \backslash N$ with $\# I=i n_{j}(N, D)-i n_{i}(N, D)$, and let $\left(N^{\prime}, D^{\prime}\right) \in \mathcal{D}$ be given by $N^{\prime}=N \cup I$ and $D^{\prime}=D \cup\left(\bigcup_{h \in I}\{(i, h),(h, i)\}\right)$. Since $i n_{i}\left(N^{\prime}, D^{\prime}\right)=i n_{j}\left(N^{\prime}, D^{\prime}\right)$ and $\operatorname{cop}_{i}\left(N^{\prime}, D^{\prime}\right)=\operatorname{cop}_{i}(N, D)>\operatorname{cop}_{j}(N, D)=\operatorname{cop}_{j}\left(N^{\prime}, D^{\prime}\right)$, it follows that out $t_{i}\left(N^{\prime}, D^{\prime}\right)>$ out $_{j}\left(N^{\prime}, D^{\prime}\right)$.

Suppose that the nodes in $I$ are labeled as $I=\left\{i_{1}, \ldots, i_{p}\right\}$, and thus $p=\# I=$ $i n_{j}(N, D)-i n_{i}(N, D)$. Further, let $I^{\prime} \subset \mathbb{N} \backslash N^{\prime}$ with $\# I^{\prime}=2 \# I$, and suppose that the nodes in $I^{\prime}$ are labeled as $I^{\prime}=\left\{i_{11}, \ldots, i_{1 p}, i_{21}, \ldots i_{2 p}\right\}$. Define $\left(N^{\prime \prime}, D^{\prime \prime}\right)$ by $N^{\prime \prime}=N^{\prime} \cup I^{\prime}$ and

$$
\left.D^{\prime \prime}=\left(D^{\prime} \backslash\left(\bigcup_{h \in I}(i, h),(h, i)\right\}\right)\right) \cup\left(\bigcup_{k=1}^{p}\left\{\left(i, i_{1 k}^{\prime}\right),\left(i_{k 1}^{\prime}, i_{k}\right),\left(i_{k}, i_{2 k}^{\prime}\right),\left(i_{2 k}^{\prime}, i\right)\right\}\right),
$$


being the directed graph where we put intermediary nodes on symmetric arcs between $i$ and the nodes in the set $I$, in both directions.

Notice that $i n_{i}\left(N^{\prime \prime}, D^{\prime \prime}\right)=i n_{j}\left(N^{\prime \prime}, D^{\prime \prime}\right)=i n_{j}(N, D)$ and out $\left(N^{\prime \prime}, D^{\prime \prime}\right)=$ out $_{i}(N, D)+$ $\# I=\operatorname{out}_{i}(N, D)+i n_{j}(N, D)-i n_{i}(N, D)>\operatorname{out}_{j}(N, D)=\operatorname{out}_{j}\left(N^{\prime \prime}, D^{\prime \prime}\right)$, where the inequality follows from $\operatorname{cop}_{i}(N, D)>\operatorname{cop}_{j}(N, D)$.

Let $\left(N^{\prime \prime}, D^{\prime \prime \prime}\right) \in \mathcal{D}$ be such that $D^{\prime \prime \prime} \subset D^{\prime \prime}$ with

$-(h, g) \in D^{\prime \prime} \backslash D^{\prime \prime \prime} \Rightarrow h=i$, and

$-\operatorname{out}_{i}\left(N^{\prime \prime}, D^{\prime \prime \prime}\right)=\operatorname{out}_{j}\left(N^{\prime \prime}, D^{\prime \prime \prime}\right)=\operatorname{out}_{j}\left(N^{\prime \prime}, D^{\prime \prime}\right)$.

Since $\left(N^{\prime \prime}, D^{\prime \prime \prime}\right)$ is as in Case A, we have that $i \sim_{\left(N^{\prime \prime}, D^{\prime \prime \prime}\right)} j$.

Since $D^{\prime \prime}$ is obtained from $D^{\prime \prime \prime}$ by adding arcs with node $i$ as predecessor, repeated application of positive responsiveness implies that $i \succ_{\left(N^{\prime \prime}, D^{\prime \prime}\right)} j$.

The intermediary property implies that the ranking between $i$ and $j$ is the same in $\left(N^{\prime}, D^{\prime}\right)$ as in $\left(N^{\prime \prime}, D^{\prime \prime}\right)$, and thus $i \succ_{\left(N^{\prime}, D^{\prime}\right)} j$.

Repeated application of sibling neutrality 2 implies that $i \succeq_{(N, D)} j$.

In a similar way, it can be shown that $j \nsucceq_{(N, D)} i$, and thus $i \succ_{(N, D)} j$.

If $i n_{i}(N, D)>i n_{j}(N, D)$, we start by taking $J \subset \mathbb{N} \backslash N$ with $\# J=i n_{i}(N, D)-i n_{j}(N, D)$, and let $\left(N^{\prime}, D^{\prime}\right) \in \mathcal{D}$ be given by $N^{\prime}=N \cup J$ and $D^{\prime}=D \cup\left(\bigcup_{h \in J}\{(j, h),(h, j)\}\right)$. (Again, $i n_{i}\left(N^{\prime}, D^{\prime}\right)=i n_{j}\left(N^{\prime}, D^{\prime}\right)$ and $\operatorname{out}_{i}\left(N^{\prime}, D^{\prime}\right)>\operatorname{out}_{j}\left(N^{\prime}, D^{\prime}\right)$.) Then, the proof follows a similar reasoning as above, but now replacing $\left(N^{\prime \prime}, D^{\prime \prime}\right)$ by the similar digraph that is obtained by putting intermediary nodes on symmetric arcs between $j$ and the nodes in the set $J$, in both directions, and defining $D^{\prime \prime \prime}$ in the same way as above.

\section{Proof of TheOREM 2}

To prove uniqueness, given Lemma 2, we still have to consider the case where not all conditions $S_{(N, D)}(i) \cap P_{(N, D)}(i)=S_{(N, D)}(i) \cap P_{(N, D)}(j)=S_{(N, D)}(j) \cap P_{(N, D)}(i)=S_{(N, D)}(j) \cap$ $P_{(N, D)}(j)=\emptyset$ and $(i, j),(j, i) \notin D$ are satisfied. This is dealt with by the intermediary property in the same way as it was done for the ranking by degree ratio in the proof of Theorem 1.

We are left to show that the ranking by Copeland score satisfies the axioms. $\succeq^{c o p}$ satisfying anonymity is obvious.

$\succeq^{c o p}$ satisfying positive responsiveness follows since a node's Copeland score is increasing if its outdegree increases, which has no positive effect for the other nodes.

$\succeq^{c o p}$ satisfying independence of irrelevant arcs follows since the Copeland score of a node only depends on the arcs with its successors and predecessors.

$\succeq^{c o p}$ satisfying sibling neutrality 2 follows since $\operatorname{cop}_{i}\left(N^{\prime}, D^{\prime}\right)=\operatorname{out}_{i}\left(N^{\prime}, D^{\prime}\right)-i n_{i}\left(N^{\prime}, D^{\prime}\right)=$ $\left.\operatorname{cout}_{i}(N, D)+1\right)-\left(i n_{i}(N, D)+1\right)=\operatorname{out}_{i}(N, D)-i n_{i}(N, D)=\operatorname{cop}_{i}(N, D)$, and $\operatorname{cop}_{j}\left(N^{\prime}, D^{\prime}\right)=$ $\operatorname{cop}_{j}(N, D)$ for all $j \in N \backslash\{i\}$, where $\left(N^{\prime}, D^{\prime}\right)$ is as given in the axiom.

$\succeq^{\text {cop }}$ satisfying the intermediary property follows since $\operatorname{cop}_{j}\left(N^{\prime}, D^{\prime}\right)=\operatorname{cop}_{j}(N, D)$ for all $j \in N$ with $\left(N^{\prime}, D^{\prime}\right)$ as given in the axiom. 


\section{A3: Proof of Theorem 3}

We prove Theorem 3 in several steps that are similar to the proof of Theorem 1.

Lemma 3. Let $(N, D) \in \mathcal{D}$ and $i, j \in N$ be such that $S_{(N, D)}(i) \cap P_{(N, D)}(i)=S_{(N, D)}(i) \cap$ $P_{(N, D)}(j)=S_{(N, D)}(j) \cap P_{(N, D)}(i)=S_{(N, D)}(j) \cap P_{(N, D)}(j)=\emptyset,(i, j),(j, i) \notin D$ and $i n_{i}(N, D) \cdot i n_{j}(N, D)>0$. If ranking method $\succeq$ satisfies the axioms of Theorem 3 , then $\left[i \succeq j\right.$ if and only if $\left.\bar{r}_{i}(N, D) \geq \bar{r}_{j}(N, D)\right]$.

Proof The proof for this case goes very similar to the proof of Lemma 1, but with modified definitions of the modified digraphs. We give the full proof for completeness. We distinguish the following cases:

\section{Case A}

Suppose that $\bar{r}_{i}(N, D)=\bar{r}_{j}(N, D)$. We will again shortly denote $\bar{r}_{i}$ and $\bar{r}_{j}$ for $\bar{r}_{i}(N, D)$, respectively $\bar{r}_{j}(N, D)$.

First, we enter a sibling of node $i\left(i n_{j}(N, D)-1\right.$ times $)$, and $j\left(i n_{i}(N, D)-1\right.$ times $)$ as in sibling neutrality 3 , in order to create symmetric positions for them.

Therefore, define $P_{i}^{\prime}, P_{j}^{\prime}, S_{i}^{\prime}, S_{j}^{\prime}, I, J \subset \mathbb{N} \backslash N$ such that each pair of these sets are disjoint, and

- \#I $=i n_{j}(N, D)-1, \# J=i n_{i}(N, D)-1$;

- \#P $P_{i}^{\prime}=\left(i n_{j}(N, D)-1\right) \cdot i n_{i}(N, D)$, and $\# P_{j}^{\prime}=\left(i n_{i}(N, D)-1\right) \cdot i n_{j}(N, D)$;

- \#S $S_{i}^{\prime}=\left(i_{j}(N, D)-1\right) \cdot$ out $_{i}(N, D)$, and $\# S_{j}^{\prime}=\left(i n_{i}(N, D)-1\right) \cdot$ out $_{j}(N, D)$.

Further, let digraph $\left(N^{\prime}, D^{\prime}\right) \in \mathcal{D}$ be given by

$$
N^{\prime}=N \cup S_{i}^{\prime} \cup S_{j}^{\prime} \cup P_{i}^{\prime} \cup P_{j}^{\prime} \cup I \cup J
$$

and

$$
D^{\prime}=D \cup\left(\bigcup_{h \in S_{i}^{\prime}}\{(i, h)\}\right) \cup\left(\bigcup_{h \in P_{i}^{\prime}}\{(h, i)\}\right) \cup\left(\bigcup_{h \in S_{j}^{\prime}}\{(j, h)\}\right) \cup\left(\bigcup_{h \in P_{j}^{\prime}}\{(h, j)\}\right)
$$

Next, similar as in the previous proofs, we delete all arcs that do not contain node $i$ or $j$, defining $\left(N^{\prime}, D^{\prime \prime}\right)$ similar as in the previous proofs with $D^{\prime \prime}$ given by

$$
D^{\prime \prime}=\left\{(h, g) \in D^{\prime} \mid\{h, g\} \cap\{i, j\} \neq \emptyset\right\} .
$$

Notice that

(i) $\# P_{\left(N^{\prime}, D^{\prime \prime}\right)}(i)=\# P_{i}^{\prime}+\# P_{(N, D)}(i)=\left(i n_{j}(N, D)-1\right) \cdot i n_{i}(N, D)+i n_{i}(N, D)=i n_{j}(N, D)$. $i n_{i}(N, D)=\left(i n_{i}(N, D)-1\right) \cdot i n_{j}(N, D)+i n_{j}(N, D)=\# P_{j}^{\prime}+\# P_{(N, D)}(j)=\# P_{\left(N^{\prime}, D^{\prime \prime}\right)}(j)$, and

(ii) $\# S_{\left(N^{\prime}, D^{\prime \prime}\right)}(i)=\# S_{(N, D)}(i)+\# S_{i}^{\prime}=\operatorname{out}_{i}(N, D)+\left(\operatorname{in}_{j}(N, D)-1\right) \cdot \operatorname{out}_{i}(N, D)=$ $\operatorname{out}_{i}(N, D) \cdot \operatorname{in}_{j}(N, D)=\operatorname{out}_{j}(N, D) \cdot i_{i}(N, D)=\operatorname{out}_{j}(N, D)+\left(i n_{i}(N, D)-1\right) \cdot$ out $_{j}(N, D)=$ $\# S_{(N, D)}(j)+\# S_{j}^{\prime}=\# S_{\left(N^{\prime}, D^{\prime \prime}\right)}(j)$, where the fourth equality follows from $\bar{r}_{i}(N, D)=$ $\bar{r}_{j}(N, D)$. Therefore, anonymity implies that $i \sim_{\left(N^{\prime}, D^{\prime \prime}\right)} j$.

Independence of irrelevant arcs implies that the ranking between $i$ and $j$ is the same in $\left(N^{\prime}, D^{\prime \prime}\right)$ as in $\left(N^{\prime}, D^{\prime}\right)$, and thus $i \sim_{\left(N^{\prime}, D^{\prime}\right)} j$. 
Repeated application of sibling neutrality 3 implies that $i \sim_{(N, D)} j$.

\section{Case B}

Suppose without loss of generality that $\bar{r}_{i}(N, D)>\bar{r}_{j}(N, D)$.

We define similar sets $S_{i}^{\prime}, S_{j}^{\prime}, P_{i}^{\prime}, P_{j}^{\prime}, I$ and $J$ as in Case A, so define $P_{i}^{\prime}, P_{j}^{\prime}, S_{i}^{\prime}, S_{j}^{\prime}, I, J \subset$ $\mathbb{N} \backslash N$ such that each pair of these sets is disjoint, and

- $\# I=i n_{j}(N, D)-1, \# J=i n_{i}(N, D)-1$;

- \#P $P_{i}^{\prime}=\left(i n_{j}(N, D)-1\right) \cdot i n_{i}(N, D)$, and $\# P_{j}^{\prime}=\left(i n_{i}(N, D)-1\right) \cdot i n_{j}(N, D)$;

- \#S $S_{i}^{\prime}=\left(i n_{j}(N, D)-1\right) \cdot$ out $_{i}(N, D)$, and $\# S_{j}^{\prime}=\left(i n_{i}(N, D)-1\right) \cdot$ out $_{j}(N, D)$.

Define digraph $\left(N^{\prime}, D^{\prime}\right)$ as in Case A (see (8) and (9)).

Similar as in Case A, let $D^{\prime \prime}$ be given by (10).

Similar as in Case A,

(i) $\# P_{\left(N^{\prime}, D^{\prime \prime}\right)}(i)=\# P_{i}^{\prime}+\# P_{(N, D)}(i)=\left(i n_{j}(N, D)-1\right) \cdot i n_{i}(N, D)+i n_{i}(N, D)=i n_{j}(N, D)$. $i n_{i}(N, D)=\left(i n_{i}(N, D)-1\right) \cdot i n_{j}(N, D)+i n_{j}(N, D)=\# P_{j}^{\prime}+\# P_{(N, D)}(j)=\# P_{\left(N^{\prime}, D^{\prime \prime}\right)}(j)$,

but now

(ii) $\# S_{\left(N^{\prime}, D^{\prime \prime}\right)}(i)=\# S_{(N, D)}(i)+\# S_{i}^{\prime}=\operatorname{out}_{i}(N, D)+\left(\operatorname{in}_{j}(N, D)-1\right) \cdot \operatorname{out}_{i}(N, D)=$ out $_{i}(N, D) \cdot \operatorname{in}_{j}(N, D)>$ out $_{j}(N, D) \cdot \operatorname{in}_{i}(N, D)=$ out $_{j}(N, D)+\left(\operatorname{in}_{i}(N, D)-1\right) \cdot \operatorname{out}_{j}(N, D)=$ $\# S_{(N, D)}(j)+\# S_{j}^{\prime}=\# S_{\left(N^{\prime}, D^{\prime \prime}\right)}(j)$, where the inequality follows from $\bar{r}_{i}(N, D)>\bar{r}_{j}(N, D)$.

Let $D^{\prime \prime \prime} \subset D^{\prime \prime}$ with $\left[(h, g) \in D^{\prime \prime} \backslash D^{\prime \prime \prime} \Rightarrow h=i\right]$, and $\# S_{\left(N^{\prime}, D^{\prime \prime \prime}\right)}(i)=\# S_{\left(N^{\prime}, D^{\prime \prime \prime}\right)}(j)=$ $\# S_{\left(N^{\prime}, D^{\prime \prime}\right)}(j)$.

Since $\left(N^{\prime}, D^{\prime \prime \prime}\right)$ is as in Case A, we have that $i \sim\left(N^{\prime}, D^{\prime \prime \prime}\right) j$.

Since $D^{\prime \prime}$ is obtained from $D^{\prime \prime \prime}$ by adding arcs with node $i$ as predecessor, repeated application of positive responsiveness implies that $i \succ_{\left(N^{\prime}, D^{\prime \prime}\right)} j$.

Independence of irrelevant arcs implies that the ranking between $i$ and $j$ is the same in $\left(N^{\prime}, D^{\prime}\right)$ as in $\left(N^{\prime}, D^{\prime \prime}\right)$, and thus $i \succ_{\left(N^{\prime}, D^{\prime}\right)} j$.

Repeated application of sibling neutrality 3 implies that $i \succeq_{(N, D)} j$.

In a similar way, it can be shown that $j \succeq_{(N, D)} i$, and thus $i \succ_{(N, D)} j$.

Lemma 4. Let $(N, D) \in \mathcal{D}$ and $i, j \in N$ be such that $S_{(N, D)}(i) \cap P_{(N, D)}(i)=S_{(N, D)}(i) \cap$ $P_{(N, D)}(j)=S_{(N, D)}(j) \cap P_{(N, D)}(i)=S_{(N, D)}(j) \cap P_{(N, D)}(j)=\emptyset,(i, j),(j, i) \notin D, i n_{i}(N, D)=$ 0 and $i n_{j}(N, D)>0$. If ranking method $\succeq$ satisfies the axioms of Theorem 3 , then $i \succ j$.

Proof This follows directly from the maximal property.

Lemma 5. Let $(N, D) \in \mathcal{D}$ and $i, j \in N$ be such that $S_{(N, D)}(i) \cap P_{(N, D)}(i)=S_{(N, D)}(i) \cap$ $P_{(N, D)}(j)=S_{(N, D)}(j) \cap P_{(N, D)}(i)=S_{(N, D)}(j) \cap P_{N, D)}(j)=\emptyset,(i, j),(j, i) \notin D, i n_{i}(N, D)=$ $i n_{j}(N, D)=0$. If ranking method $\succeq$ satisfies the axioms of Theorem 3 , then $[i \succeq j \Leftrightarrow$ out $_{i}(N, D) \geq$ out $\left._{j}(N, D)\right]$. 
Proof We distinguish two cases.

Case A Suppose that $i n_{i}(N, D)=i n_{j}(N, D)=0$ and out $_{i}(N, D)=$ out $_{j}(N, D)$.

Let $D^{\prime} \subseteq D$ be again obtained by deleting all irrelevant arcs, so $D^{\prime}=\{(h, g) \in D \mid$ $\{h, g\} \cap\{i, j\} \neq \emptyset\}$.

Anonymity implies that $i \sim_{\left(N, D^{\prime}\right)} j$.

Then, independence of irrelevant arcs implies that $i \sim_{(N, D)} j$.

Case B Suppose that $i n_{i}(N, D)=i n_{j}(N, D)=0$ and out $_{i}(N, D)>$ out $_{j}(N, D)$.

Let $D^{\prime} \subseteq D$ be such that

- $(h, g) \in D \backslash D^{\prime}$ implies that $h=i$, and

$-\operatorname{out}_{i}\left(N, D^{\prime}\right)=\operatorname{out}_{j}\left(N, D^{\prime}\right)=\operatorname{out}_{j}(N, D)$.

Since $\left(N, D^{\prime}\right)$ is as in Case A, it follows that $i \sim_{\left(N, D^{\prime}\right)} j$.

Repeated application of positive responsiveness implies that $i \succ_{(N, D)} j$.

Proof of TheOREM 3

To prove uniqueness, given Lemma's 1,4 and 5 , we still have to consider the case where not all conditions $S_{(N, D)}(i) \cap P_{(N, D)}(i)=S_{(N, D)}(i) \cap P_{N, D)}(j)=S_{(N, D)}(j) \cap P_{(N, D)}(i)=$ $S_{(N, D)}(j) \cap P_{N, D)}(j)=\emptyset$ and $(i, j),(j, i) \notin D$ are satisfied. This is dealt with by the intermediary property in a similar way as in the proof of Theorem 1.

Also, the ranking by modified degree ratio satisfying the axioms follows similarly as in the proof of Theorem 1 (notice that in sibling neutrality 3, we only consider nodes $i$ with $\left.P_{(N, D)}(i) \neq \emptyset\right)$, while $\succeq^{m d r}$ satisfying the maximal property follows by definition. 


\section{Bibliography}

A. Altman and M. Tennenholtz. Ranking systems: The PageRank axioms. In ECÕO5 Proceedings of the 6th ACM Conference on Electronic Commerce, pages 1-8. ACM, New York, 2005.

A. Altman and M. Tennenholtz. Axiomatic foundations for ranking systems. Journal of Artificial Intelligence Research, 31:473-495, 2008.

D. Bouyssou. Ranking methods based on valued preference relations: A characterization of the net flow method. European Journal of Operational Research, 60:61-67, 1992.

D. Bouyssou and T. Marchant. The $\beta$-ranking and the $\beta$-measure for directed networks: Axiomatic characterizations. Social Networks, 52:145-153, 2018.

D. Bouyssou and P. Perny. Ranking methods for valued preference relations: A characterization of a method based on leaving and entering flows. European Journal of Operational Research, 61:186-194, 1992.

S. Brin and L. Page. The anatomy of large-scale hypertextual Web search engine. Computer Networks and ISDN Systems, 30:107-117, 1998.

A. H. Copeland. A Reasonable Social Welfare Function. Mimeographed Notes, University of Michigan Seminar on Applications of Mathematics to the Social Sciences, 1951.

G. de Clippel, H. Moulin, and N. Tideman. Impartial division of a dollar. Journal of Economic Theory, 139:176-191, 2008.

G. Demange. On the influence of a ranking system. Social Choice and Welfare, 39: 431-455, 2012.

G. Demange. A ranking method based on handicaps. Theoretical Economics, 9(3):915942, 2014a.

G. Demange. Collective attention and ranking methods. Journal of Dynamics and Games, 1:17-43, 2014b.

G. Demange. Mutual rankings. Mathematical Social Sciences, 90:35-42, 2017.

Y. Du, E. Lehrer, and A. Pauzner. Competitive economy as a ranking device over networks. Games and Economic Behavior, 91:1-13, 2015.

D. Henriet. The Copeland choice function - an axiomatic characterization. Social Choice and Welfare, 2:49-63, 1985.

D. Kahneman and A. Tversky. Prospect theory: An analysis of decision under risk. Econometrica, 47:263-291, 1979.

I. Palacios-Huerta and O. Volij. The measurement of intellectual influence. Econometrica, 72:963-977, 2004.

G. Pinski and F. Narin. Citation influence for journal aggregates of scientific publications: Theory, with application to the literature of physics. Information Processing and Management, 12:297-312, 1976.

A. Rubinstein. Ranking the participants in a tournament. SIAM Journal of Applied Mathematics, 38(1):108-111, 1980.

A. K. Sen. Collective Choice and Social Welfare. North Holland Publishing Company, Amsterdam, The Netherlands, 1979.

G. Slutzki and O. Volij. Scoring of web pages and tournaments - axiomatizations. Social Choice and Welfare, 26:75-92, 2006. 
R. van den Brink and R. P. Gilles. Measuring domination in directed networks. Social Networks, 22:141-157, 2000.

R. van den Brink and R. P. Gilles. Ranking by outdegree for directed graphs. Discrete Mathematics, 271:261-270, 2003.

R. van den Brink and R. P. Gilles. The outflow ranking method for weighted directed graphs. European Journal of Operational Research, 193:484-491, 2009.

G. J. Woeginger. An axiomatic characterization of the Hirsch-index. Mathematical Social Sciences, 56:224-232, 2008. 